MATHEMATICS OF COMPUTATION

Volume 73 , Number 246 , Pages 613-635

S 0025-5718(03)01573-4

Article electronically published on June 19, 2003

\title{
LINEARLY IMPLICIT METHODS FOR NONLINEAR PARABOLIC EQUATIONS
}

\author{
GEORGIOS AKRIVIS AND MICHEL CROUZEIX
}

\begin{abstract}
We construct and analyze combinations of rational implicit and explicit multistep methods for nonlinear parabolic equations. The resulting schemes are linearly implicit and include as particular cases implicit-explicit multistep schemes as well as the combination of implicit Runge-Kutta schemes and extrapolation. An optimal condition for the stability constant is derived under which the schemes are locally stable. We establish optimal order error estimates.
\end{abstract}

\section{INTRODUCTION}

Let $T>0, u^{0} \in H$, and consider the initial value problem of seeking $u:[0, T] \rightarrow$ $D(A)$ satisfying

$$
\left\{\begin{array}{l}
u^{\prime}(t)+A u(t)=B(t, u(t)), \quad 0<t<T, \\
u(0)=u^{0}
\end{array}\right.
$$

with $A$ a positive definite, self-adjoint, linear operator on a Hilbert space $(H,(\cdot, \cdot))$ with domain $D(A)$ dense in $H$, and $B(t, \cdot): D(A) \rightarrow H, t \in[0, T]$, a (possibly) nonlinear operator. We assume that (1.1) possesses a smooth solution.

The schemes we will consider in this paper are expressed in terms of bounded rational functions $\rho_{i}, \sigma_{i}:[0, \infty] \rightarrow \mathbb{R}, i=0, \ldots, q$, with $\rho_{q}=1$ and $\sigma_{q}=0$; we assume that the functions $\sigma_{i}$ vanish at infinity, $\sigma_{i}(\infty)=0$. The implicit scheme described by the functions $\rho_{i}, i=0, \ldots, q$, will be used for the discretization of the linear part and the explicit scheme described by the functions $\sigma_{i}, i=0, \ldots, q-1$, for the discretization of the nonlinear part of the equation.

Let $N \in \mathbb{N}$, let $k:=T / N$ be the time step, and let $t^{n}:=n k, n=0, \ldots, N$. We recursively define a sequence of approximations $U^{m}$ to $u^{m}:=u\left(t^{m}\right)$ by

$$
\sum_{i=0}^{q} \rho_{i}(k A) U^{n+i}=k \sum_{i=0}^{q-1} \sigma_{i}(k A) B\left(t^{n+i}, U^{n+i}\right),
$$

assuming that starting approximations $U^{0}, \ldots, U^{q-1}$ are given.

Let $|\cdot|$ denote the norm of $H$, and introduce in $V, V:=D\left(A^{1 / 2}\right)$, the norm $\|\cdot\|$ by $\|v\|:=\left|A^{1 / 2} v\right|$. We identify $H$ with its dual, and we denote by $V^{\prime}$ the dual of $V$

Received by the editor May 2, 2001 and, in revised form, October 2, 2002.

2000 Mathematics Subject Classification. Primary 65M60, 65M12; Secondary 65L06.

Key words and phrases. Nonlinear parabolic equations, linearly implicit methods, strong $A(0)$ stability, implicit-explicit multistep schemes, polynomial order.

The work of the first author was supported in part by the Greek Secretariat for Research and Technology through the PENED Program, no 99ED 275.

(C)2003 American Mathematical Society 
and by $\|\cdot\|_{\star}$ the dual norm on $V^{\prime}$. For stability purposes, we assume that $B(t, \cdot)$ can be extended to an operator from $V$ into $V^{\prime}$, and an estimate of the form

$$
\|B(t, v)-B(t, w)\|_{\star} \leq \lambda\|v-w\|+\mu|v-w| \quad \forall v, w \in T_{u}
$$

holds in a tube $T_{u}, T_{u}:=\left\{v \in V: \min _{t}\|u(t)-v\| \leq 1\right\}$, around the solution $u$, uniformly in $t$, with the stability constant $\lambda<1$ and a constant $\mu$.

Stability assumptions. For $x \in[0, \infty]$ we introduce the polynomials $\rho(x, \cdot)$ and $\sigma(x, \cdot)$ by

$$
\rho(x, \zeta):=\sum_{i=0}^{q} \rho_{i}(x) \zeta^{i}, \quad \sigma(x, \zeta):=\sum_{i=0}^{q-1} \sigma_{i}(x) \zeta^{i} .
$$

We order the roots $\zeta_{j}(x), j=1, \ldots, q$, of $\rho(x, \cdot)$ in such a way that the functions $\zeta_{j}$ are continuous in $[0, \infty]$ and the roots $\xi_{j}:=\zeta_{j}(0), j=1, \ldots, s$, satisfy $\left|\xi_{j}\right|=1$; these unimodular roots are the principal roots of $\rho(0, \cdot)$ and the complex numbers $\lambda_{j}:=\frac{\partial_{1} \rho\left(0, \xi_{j}\right)}{\xi_{j} \partial_{2} \rho\left(0, \xi_{j}\right)}$ (with $\partial_{1}$ denoting differentiation with respect to the first variable) are the growth factors of $\xi_{j}$. We assume that the method described by the rational functions $\rho_{0}, \ldots, \rho_{q}$ is strongly $A(0)$-stable in the sense that for all $0<x \leq \infty$ and for all $j=1, \ldots, q$, there holds $\left|\zeta_{j}(x)\right|<1$, and the principal roots of $\rho(0, \cdot)$ are simple and their growth factors have positive real parts, $\operatorname{Re} \lambda_{j}>0, j=1, \ldots, s$. This definition is motivated by the definition of the strong $A(0)$-stability for multistep schemes. Depending on the particular scheme that we use for discretizing (1.1) in time, it will be essential for our analysis that $\lambda$ be appropriately small. More precisely, with

$$
K_{(\rho, \sigma)}:=\sup _{x>0} \max _{\zeta \in S_{1}}|x \sigma(x, \zeta) / \rho(x, \zeta)|,
$$

we will assume

$$
\lambda<1 / K_{(\rho, \sigma)}
$$

here $S_{1}$ denotes the unit circle in the complex plane, $S_{1}:=\{z \in \mathbb{C}:|z|=1\}$. Under our hypotheses, $K_{(\rho, \sigma)}$ is finite. We will show local stability of the scheme (1.2) provided the stability constant $\lambda$ satisfies (1.5). Let us also note that for any constant $\lambda$ exceeding the right-hand side of (1.5) we will construct examples of problems of the form (1.1) satisfying (1.3) for which the scheme (1.2) is unstable; cf. Remark 2.1. Concerning the tube $T_{u}$ on the other hand, we emphasize that it is defined in terms of the norm of $V$ for concreteness. The analysis may be modified to yield convergence under conditions analogous to (1.3) for $v$ and $w$ belonging to tubes defined in terms of other norms, not necessarily the same for both arguments; see [2].

Consistency assumptions. Let the consistency error $E^{n}, n=0, \ldots, N-q$, of the scheme (1.2) for the solution $u$ of (1.1) be given by

$$
k(I+k A)^{-1} E^{n}=\sum_{i=0}^{q} \rho_{i}(k A) u^{n+i}-k \sum_{i=0}^{q-1} \sigma_{i}(k A) B\left(t^{n+i}, u^{n+i}\right) .
$$


Let $p \geq 1$, and let functions $\varphi_{\ell}:[0, \infty) \rightarrow \mathbb{R}, \ell=0, \ldots, p$, be defined by

$$
\begin{aligned}
\varphi_{\ell}(x) & :=\sum_{i=0}^{q}\left[i^{\ell} \rho_{i}(x)-\left(\ell i^{\ell-1}+x i^{\ell}\right) \sigma_{i}(x)\right], \quad \ell=0, \ldots, p-1, \\
\varphi_{p}(x) & :=\sum_{i=0}^{q}\left[i^{p} \rho_{i}(x)-p i^{p-1} \sigma_{i}(x)\right] .
\end{aligned}
$$

Similar functions are used in the analysis of single step schemes for inhomogeneous parabolic equations in [3]; see also 11]. Applying our scheme to initial value problems for linear scalar ordinary differential equations of the form

$$
\left\{\begin{array}{l}
u^{\prime}+a u=f, \quad 0<t<T, \\
u(0)=u^{0}
\end{array}\right.
$$

with a positive constant $a$ and $f \in C^{p}[0, T]$, we see that the scheme is of order $p$, i.e., the consistency error can be estimated in the form $\left|E^{n}\right| \leq C k^{p}$ with a constant $C$ depending on the solution $u$, for any smooth function $f$, if and only if

$$
\varphi_{\ell}(x)=O\left(x^{p+1-\ell}\right) \quad \text { as } x \rightarrow 0+, \quad \ell=0, \ldots, p ;
$$

the only if part is seen by taking $u(t)=\left(t-t^{n}\right)^{\ell}$ and the corresponding $f$, and the if part is seen via Taylor expansion. In particular, from $\varphi_{0}(0)=0$ we conclude

$$
\rho_{0}(0)+\cdots+\rho_{q}(0)=0 .
$$

We say that the polynomial order of the scheme is $\tilde{p} \leq p$, or that the scheme is strictly accurate of order $\tilde{p}$, if it integrates (1.9) exactly, whenever the exact solution $u$ is a polynomial of degree at most $\tilde{p}-1$. It is easily seen that the polynomial order of our scheme is $\tilde{p}$ if and only if

$$
\varphi_{\ell}=0, \quad \ell=0, \ldots, \tilde{p}-1 .
$$

To motivate the definition of these functions and to explain the condition for the polynomial accuracy, let us introduce the notation $E_{n}(k, a, u)$ by

$$
E_{n}(k, a, u)=\sum_{i=0}^{q} \rho_{i}(k a) u\left(t^{n+i}\right)-k \sum_{i=0}^{q} \sigma_{i}(k a)\left(u^{\prime}\left(t^{n+i}\right)+a u\left(t^{n+i}\right)\right) ;
$$

then we can see that

$$
\varphi_{\ell}(x)=E_{0}\left(1, x, v_{\ell}\right) \quad \text { with } v_{\ell}(t):=t^{\ell}, \quad \text { for } \ell=0, \ldots, p-1,
$$

i.e., the polynomial order of the scheme $(1.2)$ is $\tilde{p} \leq p$ if and only if $\left(\tilde{C}_{\tilde{p}}\right)$ is satisfied. Let us also note for later use that, with $v_{\ell, q}(t):=(t-q+1)^{\ell}$ and $\varphi_{\ell, q}(x)=$ $E_{0}\left(1, x, v_{\ell, p}\right),\left(\tilde{C}_{\tilde{p}}\right)$ is clearly equivalent to

$$
\varphi_{\ell, q}=0, \quad \ell=0, \ldots, \tilde{p}-1 .
$$

To implement (1.2), for each linear factor in the denominator of the rational functions $\rho_{i}$ and $\sigma_{i}, i=0, \ldots, q$, we need to solve one linear problem at every time level. The linear problems reduce to linear systems when we also discretize in space. Therefore, from a computational point of view it would be convenient to choose the rational functions $\sigma_{i}$ such that their denominators are all the same as that of the functions $\rho_{j}$, because for each common linear factor of the denominators we have to solve one linear problem. One way of achieving this, as well as condition $\left(\tilde{C}_{p}\right)$, is 
to first choose $\hat{\rho}_{0}, \ldots, \hat{\rho}_{q}$ with $\hat{\rho}_{q}=1$ such that $\sum_{i=0}^{q} i^{j} \hat{\rho}_{i}(x)=O\left(x^{p+1-j}\right)$ as $x \rightarrow$ $0+, j=0, \ldots, p$, i.e.,

$$
\sum_{i=0}^{q} \mathrm{e}^{-i x} \hat{\rho}_{i}(x)=O\left(x^{p+1}\right) \quad \text { as } x \rightarrow 0+,
$$

and to let the corresponding scheme be strongly $A(0)$-stable and then to distinguish two cases: for $q \geq p$ we set $\rho_{i}:=\hat{\rho}_{i}, i=0, \ldots, q, \sigma_{i}:=0, i=p, \ldots, q$, and for $p>q$ we set $\rho_{i}:=0, i=0, \ldots, p-q-1, \rho_{p-q+i}:=\hat{\rho}_{i}, i=0, \ldots, q$, and then we solve the linear system

$$
\sum_{i=0}^{p-1} i^{\ell} \sigma_{i}(x)=-\frac{\ell !}{(-x)^{\ell+1}} \sum_{j=0}^{\ell} \frac{(-x)^{j}}{j !} \sum_{i=0}^{\max (p, q)} i^{j} \rho_{i}(x), \quad \ell=0, \ldots, p-1,
$$

to determine the rational functions $\sigma_{i}, i=0, \ldots, p-1$. This system is obviously uniquely solvable and the rational functions $\sigma_{i}$ are linear combinations of the righthand sides of (1.12). Hence, the only singularities of the $\sigma_{i}$ are those of the $\rho_{j}$ (which are the only singularities of the right-hand sides of (1.12)), i.e., the denominator of all $\sigma_{i}$ is the least common multiple of the denominators of the functions $\rho_{0}, \ldots, \rho_{q}$. Also, since the functions $\rho_{i}$ are bounded, the right-hand sides of (1.12) are small for large $x$, i.e., the numerator of $\sigma_{i}$ is of lower degree than its denominator; thus $\sigma_{i}(\infty)=0$.

Assuming that the order and the polynomial order of our scheme are $p$ and that the solution $u$ of (1.1) is sufficiently smooth, we shall estimate the consistency error in the form

$$
\max _{0 \leq n \leq N-q}\left\|E^{n}\right\|_{\star} \leq C k^{p} .
$$

In our main result, we shall derive optimal order error estimates in $|\cdot|$, assuming (1.5), that the order of our scheme is $p$ and its polynomial order is $p-1$, and that appropriate starting values $U^{0}, \ldots, U^{q-1}$ are given.

Let us note that the implicit-explicit multistep schemes analyzed in 1$]$ and [2] are particular cases of the schemes considered in this paper. Indeed, if we let $(\alpha, \beta)$ be a strongly $A(0)$-stable $q$-step scheme and $(\alpha, \gamma)$ be an explicit $q$-step scheme, characterized by three polynomials $\alpha, \beta$ and $\gamma$,

$$
\alpha(\zeta)=\sum_{i=0}^{q} \alpha_{i} \zeta^{i}, \quad \beta(\zeta)=\sum_{i=0}^{q} \beta_{i} \zeta^{i}, \quad \gamma(\zeta)=\sum_{i=0}^{q-1} \gamma_{i} \zeta^{i},
$$

then the corresponding implicit-explicit $(\alpha, \beta, \gamma)$ scheme for $(1.1)$ is

$$
\sum_{i=0}^{q}\left(\alpha_{i} I+k \beta_{i} A\right) U^{n+i}=k \sum_{i=0}^{q-1} \gamma_{i} B\left(t^{n+i}, U^{n+i}\right) .
$$

Now letting $\rho_{i}(x):=\left(\alpha_{i}+\beta_{i} x\right) /\left(\alpha_{q}+\beta_{q} x\right), i=0, \ldots, q$, and letting $\sigma_{i}(x):=$ $\gamma_{i} /\left(\alpha_{q}+\beta_{q} x\right), i=0, \ldots, q-1$, it is easily seen that the scheme (1.2) reduces to (1.14). Also, the stability and consistency conditions in this paper coincide in this case with those of $[2]$.

Keeling [5] has constructed and analyzed combinations of a strongly $A_{0}$-stable implicit Runge-Kutta method (IRKM) and an extrapolation scheme for the discretization of semilinear parabolic equations. These schemes, even for stronger nonlinearities, are included in the class considered in this paper; see Section 7. 
The analysis in this paper is based on the one in 2] and concerns a much wider class of methods. We also note that ideas from [3] are used in our analysis in various places.

Roughly speaking, our methods can be viewed as an extension of the Rosenbrock methods based on an inexact Jacobian; the motivation is the same as in [10]. Other classes of linearly implicit methods are constructed in [8] and [6]; they correspond to the use of approximate Jacobians. Clearly the methods based on approximate Jacobians have better stability properties and are easier to analyze. An advantage of our methods is that they avoid recomputation of these Jacobians.

An outline of the paper is as follows: Sections 2 and 3 are devoted to the local stability and consistency, respectively, of the linearly implicit scheme. Optimal order error estimates are derived in Section 4. Section 5 is devoted to error estimates for fully discrete schemes. The computation of appropriate starting approximations is addressed in Section 6. In Section 7 we shall show that Keeling's schemes can be written in the form (1.2) and that they satisfy our hypotheses.

\section{LOCAL STABILITY}

In this section we show local stability of the scheme (1.2) under the condition (1.5). We will also see that if $\lambda$ in (1.3) exceeds the right-hand side of (1.5), then for an appropriate choice of $A$ and $B$ the scheme (1.2) is unstable.

Let $U^{m}, V^{m} \in T_{u}, m=0, \ldots, N$, satisfy (1.2) and

$$
\sum_{i=0}^{q} \rho_{i}(k A) V^{n+i}=k \sum_{i=0}^{q-1} \sigma_{i}(k A) B\left(t^{n+i}, V^{n+i}\right),
$$

$n=0, \ldots, N-q$, respectively. Let $\vartheta^{m}:=U^{m}-V^{m}, b^{m}:=B\left(t^{m}, U^{m}\right)-B\left(t^{m}, V^{m}\right)$, $m=0, \ldots, N$. Subtracting (2.1) from (1.2), we obtain

$$
\sum_{i=0}^{q} \rho_{i}(k A) \vartheta^{n+i}=k \sum_{i=0}^{q-1} \sigma_{i}(k A) b^{n+i}, \quad n=0, \ldots, N-q .
$$

The rational functions $e(\ell, \cdot)$ and $f(\ell, \cdot)$ defined through the expansions

$$
(\rho(x, \zeta))^{-1}=\sum_{\ell \in \mathbb{Z}} e(\ell, x) \zeta^{-\ell}, \quad(\rho(x, \zeta))^{-1} \sigma(x, \zeta)=\sum_{\ell \in \mathbb{Z}} f(\ell, x) \zeta^{-\ell}
$$

will play a crucial role in the stability analysis. Since, for all $x \in(0, \infty]$, the modulus of all roots of $\rho(x, \cdot)$ is less than one, expansions $(2.3)$ are valid for all $\zeta$ in the exterior of the unit circle, $|\zeta| \geq 1$, and we have $e(\ell, \cdot)=0$ for $\ell \leq q-1$ and $f(\ell, \cdot)=0$ for $\ell \leq 0$. We also note that the only poles of these rational functions are the poles of $\rho_{i}, \sigma_{i}, i=0, \ldots, q$, and that they vanish at $\infty, e(\ell, \infty)=f(\ell, \infty)=0$. Thus, we can define $e(\ell, k A)$ and $f(\ell, k A)$. Let $\vartheta_{1}^{0}=0, \quad \vartheta_{1}^{n}=k \sum_{\ell=0}^{n-1} f(n-\ell, k A) b^{\ell}$. Then, in view of $(2.3)$, we have

$$
\sum_{i=0}^{q} \rho_{i}(k A) \vartheta_{1}^{n+i}=k \sum_{i=0}^{q-1} \sigma_{i}(k A) b^{n+i}, \quad n=0, \ldots, N-q .
$$

Furthermore, let $\vartheta_{2}^{n}:=\vartheta^{n}-\vartheta_{1}^{n}$. From (2.2) and (2.4) we immediately obtain

$$
\sum_{i=0}^{q} \rho_{i}(k A) \vartheta_{2}^{n+i}=0, \quad n=0, \ldots, N-q
$$


With $g_{j}(n, x)=\sum_{\ell=j+1}^{q} e(n+\ell-j, x) \rho_{\ell}(x)$, it is easily seen that $\vartheta_{2}^{n}=\sum_{j=0}^{q-1} g_{j}(n, k A) \vartheta_{2}^{j}$.

An adaptation of the techniques used in 7] (see also 9]) based on Parseval's identity allows us to prove the following result.

Lemma 2.1. There exist positive constants $K_{2}, N_{1}$ and $N_{2}$, depending only on $\rho_{i}, \sigma_{i}, i=0, \ldots, q$, such that for any $n, 0 \leq n \leq N$, the following estimates are valid:

$$
\begin{aligned}
k \sum_{\ell=0}^{n}\left\|\vartheta_{1}^{\ell}\right\|^{2} & \leq K_{(\rho, \sigma)}^{2} k \sum_{\ell=0}^{n-1}\left\|b^{\ell}\right\|_{\star}^{2}, \\
\left|\vartheta_{1}^{n}\right|^{2} & \leq K_{2} k \sum_{\ell=0}^{n-1}\left\|b^{\ell}\right\|_{\star}^{2}, \\
k \sum_{\ell=q}^{n}\left\|\vartheta_{2}^{\ell}\right\|^{2} & \leq q N_{1} \sum_{j=0}^{q-1}\left(\left|\vartheta_{2}^{j}\right|^{2}+k\left\|\vartheta_{2}^{j}\right\|^{2}\right), \\
\left|\vartheta_{2}^{n}\right| & \leq N_{2} \sum_{j=0}^{q-1}\left|\vartheta_{2}^{j}\right| .
\end{aligned}
$$

In particular, with $k_{1}(x, \zeta)=\frac{x \sigma(x, \zeta)}{\rho(x, \zeta)}, N_{2}=\max _{0 \leq j \leq q-1} \sup _{n \geq q} \sup _{x>0}\left|g_{j}(n, x)\right|$, $K_{2}=\sup _{x>0} \int_{0}^{1}\left|\frac{1}{\sqrt{x}} k_{1}\left(x, \mathrm{e}^{-2 \mathrm{i} \pi t}\right)\right|^{2} d t, \quad N_{1}=\max _{0 \leq j \leq q-1} \sup _{x>0} \int_{0}^{1} \frac{x\left|\delta_{j}\left(\mathrm{e}^{-2 \mathrm{i} \pi t}, x\right)\right|^{2}}{1+x} d t$, where $\delta_{j}(\zeta, x)=-\sum_{\ell=0}^{j} \rho_{\ell}(x) \zeta^{\ell} / \rho(x, \zeta)$.

Proof. The proof that $K_{(\rho, \sigma)}, K_{2}, N_{1}, N_{2}$ are finite is similar to analogous results in [2] and is omitted. It suffices to show the estimates for $b^{\ell}=0$ for $\ell \geq n$ and $n$ replaced by $\infty$ on the right-hand sides. We introduce $\hat{b}, \hat{\vartheta}_{1}$ and $\hat{\vartheta}_{2}$ by

$$
\hat{b}(t)=\sum_{\ell=0}^{\infty} b^{\ell} \mathrm{e}^{2 \mathrm{i} \pi \ell t}, \quad \hat{\vartheta}_{1}(t)=\sum_{\ell=0}^{\infty} \vartheta_{1}^{\ell} \mathrm{e}^{2 \mathrm{i} \pi \ell t}, \quad \hat{\vartheta}_{2}(t)=\sum_{\ell=q}^{\infty} \vartheta_{2}^{\ell} \mathrm{e}^{2 \mathrm{i} \pi \ell t}
$$

from the definition of $\vartheta_{1}$ and (2.3), we deduce

$$
\hat{\vartheta}_{1}(t)=k\left(\rho\left(k A, \mathrm{e}^{-2 \mathrm{i} \pi t}\right)\right)^{-1} \sigma\left(k A, \mathrm{e}^{-2 \mathrm{i} \pi t}\right) \hat{b}(t) .
$$

Therefore, we have $\left\|\hat{\vartheta}_{1}(t)\right\| \leq K_{(\rho, \sigma)}\|\hat{b}(t)\|_{\star}$, and, using Parseval's identity,

$$
\sum_{\ell=0}^{\infty}\left\|\vartheta_{1}^{\ell}\right\|^{2}=\int_{0}^{1}\left\|\hat{\vartheta}_{1}(t)\right\|^{2} d t \leq K_{(\rho, \sigma)}^{2} \int_{0}^{1}\|\hat{b}(t)\|_{\star}^{2} d t=K_{(\rho, \sigma)}^{2} \sum_{\ell=0}^{\infty}\left\|b^{\ell}\right\|_{\star}^{2},
$$

i.e. (2.6i) holds. For the estimate (2.6ii), we use the relation

$$
\vartheta_{1}^{n}=\int_{0}^{1} \mathrm{e}^{-2 \mathrm{i} \pi n t} \hat{\vartheta}_{1}(t) d t=\int_{0}^{1} \mathrm{e}^{-2 \mathrm{i} \pi n t} A^{-1} k_{1}\left(k A, \mathrm{e}^{-2 \mathrm{i} \pi t}\right) \hat{b}(t) d t,
$$

and we obtain

$$
\left|\vartheta_{1}^{n}\right|^{2}=\int_{0}^{1} \mathrm{e}^{-2 \mathrm{i} \pi n t}\left(\hat{b}(t), A^{-1} k_{1}\left(k A, \mathrm{e}^{2 \mathrm{i} \pi t}\right) \vartheta_{1}^{n}\right) d t,
$$


and thus

$$
\left|\vartheta_{1}^{n}\right|^{2} \leq\left(\int_{0}^{1}\|\hat{b}(t)\|_{\star}^{2} d t\right)^{1 / 2}\left(\int_{0}^{1}\left\|A^{-1} k_{1}\left(k A, \mathrm{e}^{2 \mathrm{i} \pi t}\right) \vartheta_{1}^{n}\right\|^{2} d t\right)^{1 / 2}
$$

Introducing the operator $\mathcal{A}$ by

$$
\mathcal{A}=\int_{0}^{1}(k A)^{-1} k_{1}\left(k A, \mathrm{e}^{-2 \mathrm{i} \pi t}\right) k_{1}\left(k A, \mathrm{e}^{2 \mathrm{i} \pi t}\right) d t
$$

we then have

$$
\int_{0}^{1}\left\|A^{-1} k_{1}\left(k A, \mathrm{e}^{2 \mathrm{i} \pi t}\right) \vartheta_{1}^{n}\right\|^{2} d t=k\left(\mathcal{A} \vartheta_{1}^{n}, \vartheta_{1}^{n}\right) \leq k\|\mathcal{A}\|_{\mathcal{L}(H)}\left|\vartheta_{1}^{n}\right|^{2} .
$$

Since $A$ is self-adjoint, we have

$$
\|\mathcal{A}\|_{\mathcal{L}(H)} \leq \sup _{\nu \in \operatorname{Sp}(A)} \int_{0}^{1} \frac{\left|k_{1}\left(k \nu, \mathrm{e}^{-2 \mathrm{i} \pi t}\right)\right|^{2}}{k \nu} d t \leq K_{2}
$$

with $\operatorname{Sp}(A)$ denoting the spectrum of $A$, and so we conclude that $\left|\vartheta_{1}^{n}\right|^{2} \leq$ $k K_{2} \int_{0}^{1}\|\hat{b}(t)\|_{\star}^{2} d t$ and (2.6ii) follows. In order to prove (2.7i), we first note that, in view of (2.3), an easy calculation shows that

$$
\hat{\vartheta}_{2}(t)=\sum_{j=0}^{q-1} \delta_{j}\left(\mathrm{e}^{-2 \mathrm{i} \pi t}, k A\right) \vartheta_{2}^{j} \mathrm{e}^{2 \mathrm{i} \pi j t}
$$

cf. 2]. Furthermore, as in the proof of (2.6ii),

$$
k \int_{0}^{1}\left\|\delta_{j}\left(\mathrm{e}^{-2 \mathrm{i} \pi t}, k A\right) \vartheta_{2}^{j} \mathrm{e}^{2 \mathrm{i} \pi j t}\right\|^{2} d t \leq N_{1}\left(\left|\vartheta_{2}^{j}\right|^{2}+k\left\|\vartheta_{2}^{j}\right\|^{2}\right),
$$

and, therefore,

$$
k \int_{0}^{1}\left\|\hat{\vartheta}_{2}(t)\right\|^{2} d t \leq q N_{1} \sum_{j=0}^{q-1}\left(\left|\vartheta_{2}^{j}\right|^{2}+k\left\|\vartheta_{2}^{j}\right\|^{2}\right)
$$

which immediately yields (2.7i). The estimate (2.7ii) is obvious.

In Theorem 2.1 we will estimate $\vartheta^{n}$ in terms of $\vartheta^{0}, \ldots, \vartheta^{q-1}$. Part of $\vartheta^{n}$, namely $\vartheta_{2}^{n}$, will be estimated in terms of $\vartheta^{0}, \ldots, \vartheta^{q-1}$ in the following lemma.

Lemma 2.2. There exists a constant $C$ such that, for $n=0, \ldots, N$,

$$
\left|\vartheta_{2}^{n}\right|^{2}+k \sum_{\ell=0}^{n}\left\|\vartheta_{2}^{\ell}\right\|^{2} \leq C \sum_{j=0}^{q-1}\left(\left|\vartheta^{j}\right|^{2}+k\left\|\vartheta^{j}\right\|^{2}\right)
$$

Proof. Obviously, we have $\vartheta_{2}^{j}=\vartheta^{j}-k \sum_{\ell=0}^{j-1} f(j-\ell, k A) b^{\ell}, j=0, \ldots, q-1$. Therefore

$$
\left|\vartheta_{2}^{j}\right| \leq\left|\vartheta^{j}\right|+\sqrt{k} \sum_{\ell=0}^{j-1} m_{j-\ell}\left\|b^{\ell}\right\|_{\star}, \quad \text { and } \quad\left\|\vartheta_{2}^{j}\right\| \leq\left\|\vartheta^{j}\right\|+\sum_{\ell=0}^{j-1} n_{j-\ell}\left\|b^{\ell}\right\|_{\star},
$$

with $m_{\ell}=\sup _{x>0}|\sqrt{x} f(\ell, x)|$ and $n_{\ell}=\sup _{x>0}|x f(\ell, x)|$. Then (2.8) follows from (2.7) and (1.3).

The main result in this section, the local stability of the scheme (1.2), is given in the following theorem: 
Theorem 2.1. Let $U^{m}, V^{m} \in T_{u}$ satisfy (1.2) and (2.1), respectively. Then, with $\vartheta^{m}=U^{m}-V^{m}$, we have the local stability estimate

$$
\left|\vartheta^{n}\right|^{2}+k \sum_{\ell=0}^{n}\left\|\vartheta^{\ell}\right\|^{2} \leq C \mathrm{e}^{c \mu^{2} t^{n}} \sum_{j=0}^{q-1}\left(\left|\vartheta^{j}\right|^{2}+k\left\|\vartheta^{j}\right\|^{2}\right),
$$

$n=q-1, \ldots, N$, with constants $C$ and $c$ independent of $U^{m}, V^{m}$ and $k$.

Proof. In view of (1.3) and Minkowski's inequality we have

$$
\left(k \sum_{\ell=0}^{n-1}\left\|b^{\ell}\right\|_{\star}^{2}\right)^{1 / 2} \leq\left(k \sum_{\ell=0}^{n-1}\left(\lambda\left\|\vartheta^{\ell}\right\|+\mu\left|\vartheta^{\ell}\right|\right)^{2}\right)^{1 / 2} \leq \lambda a_{n-1}+\mu d_{n-1}+e_{n-1}
$$

with

$$
a_{n}=\left(k \sum_{\ell=0}^{n}\left\|\vartheta_{1}^{\ell}\right\|^{2}\right)^{1 / 2}, \quad d_{n}=\left(k \sum_{\ell=0}^{n}\left|\vartheta_{1}^{\ell}\right|^{2}\right)^{1 / 2},
$$

and

$$
e_{n}=\left(k \sum_{\ell=0}^{n}\left(\lambda\left\|\vartheta_{2}^{\ell}\right\|+\mu\left|\vartheta_{2}^{\ell}\right|\right)^{2}\right)^{1 / 2} .
$$

Thus, (2.6i) and (2.6ii) yield, for $n \geq 1$,

$$
a_{n} \leq K_{(\rho, \sigma)}\left(\lambda a_{n-1}+\mu d_{n-1}+e_{n-1}\right) \leq K_{(\rho, \sigma)}\left(\lambda a_{n}+\mu d_{n-1}+e_{n-1}\right),
$$

and

$$
\frac{d_{n}^{2}-d_{n-1}^{2}}{k} \leq K_{2}\left(\lambda a_{n}+\mu d_{n-1}+e_{n-1}\right)^{2}
$$

therefore, in view of (1.5), we have $\lambda K_{(\rho, \sigma)}<1$ and

$$
\frac{d_{n}^{2}-d_{n-1}^{2}}{k} \leq K_{2}\left(\frac{\mu d_{n-1}+e_{n-1}}{1-\lambda K_{(\rho, \sigma)}}\right)^{2} \leq 2 c\left(\mu^{2} d_{n-1}^{2}+e_{n-1}^{2}\right),
$$

with $c=\frac{K_{2}}{\left(1-\lambda K_{(\rho, \sigma)}\right)^{2}}$. Hence, we deduce (note that $d_{0}=0$ )

$$
d_{n}^{2} \leq 2 c k \sum_{\ell=0}^{n-1} \mathrm{e}^{2 c \mu^{2}\left(t^{n-1}-t^{\ell}\right)} e_{\ell}^{2} \leq 2 c k \frac{\mathrm{e}^{2 c \mu^{2} t^{n}}-1}{\mathrm{e}^{2 c \mu^{2} k}-1} e_{n-1}^{2} \leq \frac{\mathrm{e}^{2 c \mu^{2} t^{n}}-1}{\mu^{2}} e_{n-1}^{2} .
$$

Thus, we have $\mu d_{n} \leq \mathrm{e}^{c \mu^{2} t^{n}} e_{n-1}$ and

$$
\begin{gathered}
a_{n} \leq \frac{K_{(\rho, \sigma)}}{1-K_{(\rho, \sigma)} \lambda}\left(1+\mathrm{e}^{c \mu^{2} t^{n}}\right) e_{n-1}, \\
\left|\vartheta_{1}^{n}\right| \leq \sqrt{c}\left(\mu d_{n-1}+e_{n-1}\right) \leq \sqrt{c}\left(1+\mathrm{e}^{c \mu^{2} t^{n}}\right) e_{n-1} .
\end{gathered}
$$

Now, (2.10) and (2.8) yield

$$
\left|\vartheta_{1}^{n}\right|^{2}+k \sum_{\ell=0}^{n}\left\|\vartheta_{1}^{\ell}\right\|^{2} \leq C \mathrm{e}^{c \mu^{2} t^{n}} \sum_{j=0}^{q-1}\left(\left|\vartheta^{j}\right|^{2}+k\left\|\vartheta^{j}\right\|^{2}\right) .
$$

From (2.11) and (2.8) we easily obtain (2.9) and the proof is complete. 
Remark 2.1. The condition (1.5) is sharp in the sense that, for any constant $\lambda$ exceeding the right-hand side of (1.5), we can give examples of operators $A$ and $B$ satisfying (1.3) for which the scheme (1.2) is unstable. Indeed, assume that $\lambda K_{(\rho, \sigma)}>$ 1. In view of this hypothesis and the fact that $\lim _{|\zeta| \rightarrow \infty}[x \sigma(x, \zeta) / \rho(x, \zeta)]=0$, there exists a positive $x$ and a $\zeta \in \mathbb{C}$ with $|\zeta|>1$ satisfying $|\lambda x \sigma(x, \zeta) / \rho(x, \zeta)|=1$; therefore, there exists a $\Theta \in \mathbb{R}$ such that

$$
\sum_{i=0}^{q} \rho_{i}(x) \zeta^{i}=\lambda x \mathrm{e}^{\mathrm{i} \Theta} \sum_{i=0}^{q-1} \sigma_{i}(x) \zeta^{i} .
$$

Then choosing $B(t, u):=\lambda \mathrm{e}^{\mathrm{i} \Theta} A u$, it is easily seen that the scheme is unstable; see Remark 2.3 in [2].

\section{Consistency}

In this section we will derive an optimal order estimate for the consistency error $E^{n}$ (see (3.5) below), assuming that the order and the polynomial order of the scheme are $p$. We will also derive some preliminary consistency estimates for polynomial order $p-1$ which will be used in Section 4 to establish optimal order error estimates.

Letting

$$
\begin{aligned}
& E_{1}^{n}:=\sum_{\ell=0}^{p} \frac{k^{\ell}}{\ell !} \varphi_{\ell}(k A) u^{(\ell)}\left(t^{n}\right) \\
& E_{2}^{n}:=\frac{1}{p !} \sum_{i=0}^{q} \rho_{i}(k A) \int_{t^{n}}^{t^{n+i}}\left(t^{n+i}-s\right)^{p} u^{(p+1)}(s) d s \\
& E_{3}^{n}:=-\frac{k}{(p-1) !} \sum_{i=0}^{q-1} \sigma_{i}(k A) \int_{t^{n}}^{t^{n+i}}\left(t^{n+i}-s\right)^{p-1}\left(u^{(p+1)}+A u^{(p)}\right)(s) d s
\end{aligned}
$$

and using Taylor expansion on the right-hand side of (1.6), we easily see that

$$
k(I+k A)^{-1} E^{n}=E_{1}^{n}+E_{2}^{n}+E_{3}^{n} .
$$

Assume now that the order and the polynomial order of our scheme are $p$; see $\left(\tilde{C}_{\tilde{p}}\right)$. Then, in view of (3.1a), $E_{1}^{n}=k^{p} \varphi_{p}(k A) u^{(p)}\left(t^{n}\right) / p$ !, i.e.,

$$
(I+k A) E_{1}^{n}=\frac{k^{p+1}}{p !}\left[(k A)^{-1} \varphi_{p}(k A)+\varphi_{p}(k A)\right] A u^{(p)}\left(t^{n}\right) .
$$

Now using the fact that $\varphi_{p}$ and $\tilde{\varphi}_{p}, \tilde{\varphi}_{p}(x):=\varphi_{p}(x) / x$, are bounded, we easily conclude

$$
\left\|(I+k A) E_{1}^{n}\right\|_{\star} \leq C k^{p+1}\left\|u^{(p)}\left(t^{n}\right)\right\| .
$$

Similarly, using the boundedness of $\rho_{i}, \sigma_{i}$ and $\hat{\sigma}_{i}, \hat{\sigma}_{i}(x):=x \sigma_{i}(x)$, we easily obtain

$$
\begin{aligned}
& \left\|(I+k A) E_{2}^{n}\right\|_{\star} \leq C k^{p} \int_{t^{n}}^{t^{n+q}}\left(\left\|u^{(p+1)}(s)\right\|_{\star}+k\left\|u^{(p+1)}(s)\right\|\right) d s, \\
& \left\|(I+k A) E_{3}^{n}\right\|_{\star} \leq C k^{p} \int_{t^{n}}^{t^{n+q-1}}\left(\left\|u^{(p+1)}(s)\right\|_{\star}+\left\|u^{(p)}(s)\right\|\right) d s .
\end{aligned}
$$


From (3.4) and (3.2) we immediately obtain the desired consistency estimate

$$
\max _{0 \leq n \leq N-q}\left\|E^{n}\right\|_{\star} \leq C k^{p}
$$

Remark 3.1. The case of polynomial order less than $p$ can be treated as well, but to obtain optimal order error estimates some compatibility conditions would be required; without such conditions order reduction would occur; cf. [3] and [4. However, if the polynomial order is $p-1$, using the fact that the function $\eta$,

$$
\eta(x)=\varphi_{p-1}(x) /[x \rho(x, 1)],
$$

is bounded in $[0, \infty]$, we will prove in Theorem 4.2 optimal order error estimates without any compatibility conditions. First of all, let us note that $\eta$ is bounded in a neighborhood of 0 since $\rho(0,1)=0, \partial_{2} \rho(0,1) \neq 0$ and $\varphi_{p-1}(x)=O\left(x^{2}\right)$; it is also bounded in $[c, \infty]$ for any positive $c$, since $\rho(\cdot, 1)$ is there uniformly bounded away from 0 . As a preliminary result for Theorem 4.2 , let us note that in this case, according to (3.1a), there is an additional term to $E_{1}^{n}$ which can be written as

$$
\begin{aligned}
& \frac{k^{p-1}}{(p-1) !} \varphi_{p-1}(k A) u^{(p-1)}\left(t^{n}\right) \\
& \quad=\frac{k^{p}}{(p-1) !} \eta(k A) \rho(k A, 1) A u^{(p-1)}\left(t^{n}\right) \\
& \quad=k(I+k A)^{-1} \tilde{E}^{n}+\frac{k^{p}}{(p-1) !} \eta(k A) \sum_{i=0}^{q} \rho_{i}(k A) A\left(u^{(p-1)}\left(t^{n}\right)-u^{(p-1)}\left(t^{n+i}\right)\right),
\end{aligned}
$$

with $\tilde{E}^{n}$ such that

$$
k(I+k A)^{-1} \tilde{E}^{n}=\frac{k^{p}}{(p-1) !} \eta(k A) \sum_{i=0}^{q} \rho_{i}(k A) A u^{(p-1)}\left(t^{n+i}\right) .
$$

Thus in this case, since $\tilde{\eta}, \tilde{\eta}(x):=(1+x) \eta(x)$, is bounded, (3.5) is replaced by

$$
\max _{0 \leq n \leq N-q}\left\|E^{n}-\tilde{E}^{n}\right\|_{\star} \leq C k^{p} .
$$

\section{ERror estimates}

In this section we assume (1.5), that the order of our scheme is $p$, and that its polynomial order is $p-1$, and we shall derive optimal order error estimates. In our first result, Theorem 4.1, we will assume polynomial order $p$, and in our main result, Theorem 4.2, we relax this condition to polynomial order $p-1$.

We note that we will use similar notation to that in Section 2 ; however several quantities, like $\vartheta^{m}$ and $b^{m}$, do not coincide with those in Section 2.

Let $\vartheta^{m}:=u^{m}-U^{m}, b^{m}:=B\left(t^{m}, u^{m}\right)-B\left(t^{m}, U^{m}\right), m=0, \ldots, N$. Subtracting (1.2) from (1.6), we obtain

$$
\sum_{i=0}^{q} \rho_{i}(k A) \vartheta^{n+i}=k \sum_{j=0}^{q-1} \sigma_{j}(k A) b^{n+j}+k(I+k A)^{-1} E^{n},
$$


$n=0, \ldots, N-q$. Let

$$
\begin{aligned}
& \vartheta_{1}^{0}=0, \quad \vartheta_{1}^{n}=k \sum_{\ell=0}^{n-1} f(n-\ell, k A) b^{\ell}, \\
& \vartheta_{3}^{n}=k \sum_{\ell=0}^{n-q} e(n-\ell, k A)(I+k A)^{-1} E^{\ell} .
\end{aligned}
$$

It is then easily seen that $\vartheta_{2}^{n}:=\vartheta^{n}-\vartheta_{1}^{n}-\vartheta_{3}^{n}$ satisfies

$$
\sum_{i=0}^{q} \rho_{i}(k A) \vartheta_{2}^{n+i}=0, \quad n=0, \ldots, N-q
$$

cf. (2.5). Now $\vartheta_{3}^{j}=0$ for $j \leq q-1$; therefore $\vartheta_{2}^{0}, \ldots, \vartheta_{2}^{q-1}$, and thus all $\vartheta_{2}^{n}$, depend only on the initial entries $u^{0}, \ldots, u^{q-1}, U^{0}, \ldots, U^{q-1}$.

The quantities $\vartheta_{1}^{n}, \vartheta_{2}^{n}$ can be estimated as in Lemma 2.1. Similarly, for $\vartheta_{3}^{n}$ we have

$$
\begin{aligned}
k \sum_{\ell=0}^{n}\left\|\vartheta_{3}^{\ell}\right\|^{2} & \leq M_{1}^{2} k \sum_{\ell=0}^{n-q}\left\|E^{\ell}\right\|_{\star}^{2}, \\
\left|\vartheta_{3}^{n}\right|^{2} & \leq M_{2} k \sum_{\ell=0}^{n-q}\left\|E^{\ell}\right\|_{\star}^{2},
\end{aligned}
$$

with $M_{1}=\sup _{x>0} \max _{\zeta \in S_{1}}\left|\frac{x}{(1+x) \rho(x, \zeta)}\right|$ and $M_{2}=\sup _{x>0} \int_{0}^{1}\left|\frac{\sqrt{x}}{(1+x) \rho\left(x, \mathrm{e}^{-2 \mathrm{i} \pi t}\right)}\right|^{2} d t$. As in [2] we can see that $M_{1}$ and $M_{2}$ are finite.

In our main results, Theorems 4.1 and 4.2 , we will need to estimate $\vartheta^{n}$. Part of it, namely $\vartheta_{2}^{n}+\vartheta_{3}^{n}$, can be estimated in terms of $\vartheta^{0}, \ldots, \vartheta^{q-1}$ and the consistency errors $E^{0}, \ldots, E^{N-q}$. This result follows by combining Lemma 2.2 and (4.3).

Lemma 4.1. There exists a constant $C$ such that, for $n=0, \ldots, N$,

$$
\left|\vartheta^{n}-\vartheta_{1}^{n}\right|^{2}+k \sum_{\ell=0}^{n}\left\|\vartheta^{\ell}-\vartheta_{1}^{\ell}\right\|^{2} \leq C\left\{\sum_{j=0}^{q-1}\left(\left|\vartheta^{j}\right|^{2}+k\left\|\vartheta^{j}\right\|^{2}\right)+k \sum_{\ell=0}^{n-q}\left\|E^{\ell}\right\|_{\star}^{2}\right\} .
$$

In the following theorem we establish optimal order error estimates, assuming polynomial order $p$; this condition will be relaxed in Theorem 4.2.

Theorem 4.1. Let the order and the polynomial order of the scheme be p. Assume we are given starting approximations $U^{0}, U^{1}, \ldots, U^{q-1} \in V$ to $u^{0}, \ldots, u^{q-1}$ such that

$$
\max _{0 \leq j \leq q-1}\left(\left|u^{j}-U^{j}\right|+k^{1 / 2}\left\|u^{j}-U^{j}\right\|\right) \leq C k^{p} .
$$

Let $U^{n} \in V, n=q, \ldots, N$, be recursively defined by (1.2). Let $\vartheta^{n}=u^{n}-U^{n}, n=$ $0, \ldots, N$. Then, there exist constants $C$ and $c$, independent of $k$ and $n$, such that, for $k$ sufficiently small,

$$
\left|\vartheta^{n}\right|^{2}+k \sum_{\ell=0}^{n}\left\|\vartheta^{\ell}\right\|^{2} \leq C \mathrm{e}^{c \mu^{2} t^{n}}\left\{\sum_{j=0}^{q-1}\left(\left|\vartheta^{j}\right|^{2}+k\left\|\vartheta^{j}\right\|^{2}\right)+k \sum_{\ell=0}^{n-q}\left\|E^{\ell}\right\|_{\star}^{2}\right\},
$$

$n=q-1, \ldots, N$, and

$$
\max _{0 \leq n \leq N}\left|u\left(t^{n}\right)-U^{n}\right| \leq C k^{p}
$$


Proof. In view of (4.5) and (3.5), it is easily seen that (4.7) follows from (4.6). Thus, it remains to prove (4.6). According to (4.5) and (3.5), there exists a constant $C_{\star}$ such that the right-hand side of (4.6) can be estimated by $C_{\star}^{2} k^{2 p}$,

$$
C \mathrm{e}^{c \mu^{2} T}\left\{\sum_{j=0}^{q-1}\left(\left|\vartheta^{j}\right|^{2}+k\left\|\vartheta^{j}\right\|^{2}\right)+k \sum_{\ell=0}^{N-q}\left\|E^{\ell}\right\|_{\star}^{2}\right\} \leq C_{\star}^{2} k^{2 p} .
$$

The estimate (4.6) is obviously valid for $n=q-1$. Assume that it holds for $q-1, \ldots, n-1, q \leq n \leq N$. Then, according to (4.8) and the induction hypothesis, we have, for $k$ small enough,

$$
\max _{0 \leq j \leq n-1}\left\|\vartheta^{j}\right\| \leq C_{\star} k^{p-1 / 2} \leq 1 / 2,
$$

and thus $U^{j} \in T_{u}, j=0, \ldots, n-1$. It is then easily seen (cf. the derivation of (2.11)) that

$$
\left|\vartheta_{1}^{n}\right|^{2}+k \sum_{\ell=0}^{n}\left\|\vartheta_{1}^{\ell}\right\|^{2} \leq C \mathrm{e}^{2 c \mu^{2} t^{n}} \sum_{j=0}^{q-1}\left(\left|\vartheta^{j}\right|^{2}+k\left\|\vartheta^{j}\right\|^{2}\right) .
$$

From (4.4) and (4.10) it easily follows that (4.6) holds for $n$ as well, and the proof is complete.

The main result in this section is given in the following theorem:

Theorem 4.2. Let the polynomial order of the scheme be $p-1$ and all other conditions of Theorem 4.1 be satisfied. Then the estimates of Theorem 4.1 are valid.

Proof. We let $\vartheta_{1}^{n}, \vartheta_{2}^{n}$ be as before and split $\vartheta_{3}^{n}$ in two parts, $\hat{\vartheta}_{3}^{n}$ and $\tilde{\vartheta}_{3}^{n}$, with

$$
\begin{aligned}
& \hat{\vartheta}_{3}^{n}:=k \sum_{\ell=0}^{n-q} e(n-\ell, k A)(I+k A)^{-1}\left(E^{\ell}-\tilde{E}^{\ell}\right), \\
& \tilde{\vartheta}_{3}^{n}:=k \sum_{\ell=0}^{n-q} e(n-\ell, k A)(I+k A)^{-1} \tilde{E}^{\ell} .
\end{aligned}
$$

In view of (4.3) and (3.7), $\hat{\vartheta}_{3}^{n}$ can be easily estimated. Furthermore, using (2.3), we have

$$
\begin{aligned}
\sum_{\ell=0}^{n-q} e(n-\ell, x) \sum_{i=0}^{q} \rho_{i}(x) z^{\ell+i} & \\
= & \sum_{i=0}^{q-1} g_{q-1-i}(q+i, x) z^{n-i}-\sum_{i=0}^{q-1} g_{q-1-i}(n-i, x) z^{i},
\end{aligned}
$$

and we deduce

$$
\begin{aligned}
\tilde{\vartheta}_{3}^{n}=\frac{k^{p}}{(p-1) !} \eta(k A)\left(\sum_{i=0}^{q-1} g_{q-1-i}(q+i, k A) A u^{(p-1)}\left(t^{n-i}\right)\right. \\
\left.-\sum_{i=0}^{q-1} g_{q-1-i}(n-i, k A) A u^{(p-1)}\left(t^{i}\right)\right),
\end{aligned}
$$


and thus easily, in view also of the fact that $\tilde{\eta}, \tilde{\eta}(x)=(1+x) \eta(x)$, is bounded,

$$
\left|\tilde{\vartheta}_{3}^{n}\right|^{2}+k \sum_{\ell=0}^{n}\left\|\tilde{\vartheta}_{3}^{\ell}\right\|^{2} \leq C k^{2 p}
$$

Combined with the other estimates, this shows that the results of Theorem 4.1 are valid also for polynomial order $p-1$.

Remark 4.1. The constants in this and previous sections as well as conditions like " $k$ sufficiently small" do not directly depend on the particular choice of the operators $A$ and $B$; they only depend on $\lambda, \mu$, the discretization scheme and on various norms of the solution $u$. This fact will play a crucial role in the analysis of fully discrete schemes in the next section.

\section{FULLY DisCRETE SCHEMES}

In this section we establish optimal order error estimates for fully discrete schemes assuming that the order and the polynomial order of the scheme are $p$ and $p-1$, respectively.

For the space discretization we shall use a family $V_{h}, 0<h<1$, of finite dimensional subspaces of $V$. In this section the following discrete operators will play an essential role: Define $P_{o}: V^{\prime} \rightarrow V_{h}, A_{h}: V \rightarrow V_{h}$, and the nonlinear operators $B_{h}(t, \cdot): V \rightarrow V_{h}$ by

$$
\begin{aligned}
\left(P_{o} v, \chi\right) & =(v, \chi) & & \forall \chi \in V_{h}, \\
\left(A_{h} \varphi, \chi\right) & =(A \varphi, \chi) & & \forall \chi \in V_{h}, \\
\left(B_{h}(t, \varphi), \chi\right) & =(B(t, \varphi), \chi) & & \forall \chi \in V_{h} .
\end{aligned}
$$

The semidiscrete problem corresponding to (1.1) is to seek a function $u_{h}, u_{h}(t) \in V_{h}$, satisfying

$$
\left\{\begin{array}{l}
u_{h}^{\prime}(t)+A_{h} u_{h}(t)=B_{h}\left(t, u_{h}(t)\right), \quad 0<t<T, \\
u_{h}(0)=u_{h}^{0},
\end{array}\right.
$$

with $u_{h}^{0} \in V_{h}$ a given approximation to $u^{0}$.

To construct a fully discrete scheme, we discretize (5.1) in time. Assuming that starting approximations $U^{0}, \ldots, U^{q-1} \in V_{h}$ to $u^{0}, \ldots, u^{q-1}$ are given, we define recursively a sequence of approximations $U^{m} \in V_{h}$ to $u^{m}:=u\left(t^{m}\right)$ by

$$
\sum_{i=0}^{q} \rho_{i}\left(k A_{h}\right) U^{n+i}=k \sum_{i=0}^{q-1} \sigma_{i}\left(k A_{h}\right) B_{h}\left(t^{n+i}, U^{n+i}\right) .
$$

Let $B(t, \cdot): V \rightarrow V^{\prime}$ be differentiable, and assume that the linear operator $M(t)$, $M(t):=A-B^{\prime}(t, u(t))+\kappa I$, is uniformly positive definite, for an appropriate constant $\kappa$. We introduce the "elliptic" projection $R_{h}(t): V \rightarrow V_{h}, t \in[0, T]$, by

$$
P_{o} M(t) R_{h}(t) v=P_{o} M(t) v
$$

and we refer to 2] for motivation of this definition. We will show consistency of the semidiscrete equation for $R_{h}(t) u(t)$; to this end we shall use approximation properties of the elliptic projection operator $R_{h}(t)$. We let $W(t):=R_{h}(t) u(t)$, and we assume that $R_{h}(t)$ satisfies the estimates

$$
|u(t)-W(t)|+h^{d / 2}\|u(t)-W(t)\| \leq C h^{r},
$$




$$
\left|\frac{d}{d t}[u(t)-W(t)]\right| \leq C h^{r}
$$

with two integers $r$ and $d, 2 \leq d \leq r$. We further assume that

$$
\left\|\frac{d^{j} W}{d t^{j}}(t)\right\| \leq C, \quad j=1, \ldots, p+1 .
$$

For consistency purposes with respect to the space discretization, we assume for the nonlinear part the estimate

$$
\left\|B(t, u(t))-B(t, W(t))-B^{\prime}(t, u(t))(u(t)-W(t))\right\|_{\star} \leq C h^{r} .
$$

Let $E_{h}(t) \in V_{h}$ denote the consistency error of the semidiscrete equation (5.1) for $W$,

$$
E_{h}(t):=W^{\prime}(t)+A_{h} W(t)-B_{h}(t, W(t)), \quad 0 \leq t \leq T .
$$

From the definition of $W$ we easily conclude

$$
\left(A_{h} W(t), \chi\right)=\left(A u(t)-\left[B^{\prime}(t, u(t))-\kappa I\right](u(t)-W(t)), \chi\right) \quad \forall \chi \in V_{h} .
$$

Therefore, using (1.1),

$$
\begin{aligned}
E_{h}(t)= & W^{\prime}(t)-P_{o} u^{\prime}(t)+\kappa\left[P_{o} u(t)-W(t)\right] \\
& +P_{o}\left[B(t, u(t))-B(t, W(t))-B^{\prime}(t, u(t))(u(t)-W(t))\right],
\end{aligned}
$$

and, in view of (5.3), (5.4) and (5.6), we easily obtain the following optimal order estimate for the consistency error $E_{h}$,

$$
\max _{0 \leq t \leq T}\left\|E_{h}(t)\right\|_{\star} \leq C h^{r} .
$$

The main result in this paper is given in the following theorem:

Theorem 5.1. Let the order and the polynomial order of the scheme be $p$ and $p-1$, respectively. Assume we are given starting approximations $U^{0}, U^{1}, \ldots, U^{q-1} \in V_{h}$ to $u^{0}, \ldots, u^{q-1}$ such that

$$
\max _{0 \leq j \leq q-1}\left(\left|W^{j}-U^{j}\right|+k^{1 / 2}\left\|W^{j}-U^{j}\right\|\right) \leq C\left(k^{p}+h^{r}\right) .
$$

Let $U^{n} \in V_{h}, n=q, \ldots, N$, be recursively defined by (5.2). Then, there exists a constant $C$, independent of $k$ and $h$, such that, for $k$ and $h^{2 r} k^{-1}$ sufficiently small,

$$
\max _{0 \leq n \leq N}\left|u\left(t^{n}\right)-U^{n}\right| \leq C\left(k^{p}+h^{r}\right) .
$$

Proof. Let $\rho^{n}:=u^{n}-W^{n}, n=0, \ldots, N$, with $u^{n}:=u\left(t^{n}\right)$ and $W^{n}:=W\left(t^{n}\right)$. In view of (5.3), we have

$$
\max _{0 \leq n \leq N}\left|\rho^{n}\right| \leq C h^{r}
$$

Obviously $\tilde{B}(t, v):=B(t, v)+E_{h}(t)$ (cf. (5.7)) satisfies (1.3) with the same constants $\lambda$ and $\mu$. Now let $\tilde{W}^{j}:=W^{j}, j=0, \ldots, q-1$, and define $\tilde{W}^{n}, n=q, \ldots, N$, by applying the time discretization scheme to the equation (5.7), i.e., by

$$
\sum_{i=0}^{q} \rho_{i}\left(k A_{h}\right) \tilde{W}^{n+i}=k \sum_{i=0}^{q-1} \sigma_{i}\left(k A_{h}\right) \tilde{B}_{h}\left(t^{n+i}, \tilde{W}^{n+i}\right),
$$


with $\tilde{B}_{h}(t, v)=B_{h}(t, v)+E_{h}(t)$. Then, according to Theorem 4.2 , and in view of (3.4) and (5.5),

$$
\max _{0 \leq n \leq N}\left|W^{n}-\tilde{W}^{n}\right| \leq C k^{p} .
$$

In view of (5.12) and (5.14), it remains to estimate $\vartheta^{n}:=\tilde{W}^{n}-U^{n}$. Subtracting (5.2) from (5.13), we obtain

$$
\begin{aligned}
\sum_{i=0}^{q} \rho_{i}\left(k A_{h}\right) \vartheta^{n+i}= & k \sum_{i=0}^{q-1} \sigma_{i}\left(k A_{h}\right)\left[B_{h}\left(t^{n+i}, \tilde{W}^{n+i}\right)-B_{h}\left(t^{n+i}, U^{n+i}\right)\right] \\
& +k \sum_{i=0}^{q-1} \sigma_{i}\left(k A_{h}\right) E_{h}\left(t^{n+i}\right) .
\end{aligned}
$$

Now using the boundedness of $\sigma_{i}$ and (4.6), we get

$$
\left|\vartheta^{n}\right|^{2}+k \sum_{\ell=0}^{n}\left\|\vartheta^{\ell}\right\|^{2} \leq C \mathrm{e}^{c \mu^{2} t^{n}}\left\{\sum_{j=0}^{q-1}\left(\left|\vartheta^{j}\right|^{2}+k\left\|\vartheta^{j}\right\|^{2}\right)+k \sum_{\ell=0}^{n-q}\left\|E_{h}\left(t^{\ell}\right)\right\|_{\star}^{2}\right\} .
$$

From this estimate, in view of (5.9) and our condition on the starting approximations, we easily conclude

$$
\max _{0 \leq n \leq N}\left|\tilde{W}^{n}-U^{n}\right| \leq C\left(k^{p}+h^{r}\right) .
$$

Let us note that it is in the derivation of (5.16) and (5.17) where we need the mesh condition " $h^{2 r} k^{-1}$ sufficiently small"; this is due to the fact that the analog of (4.9) now reads

$$
\max _{0 \leq j \leq n-1}\left\|\vartheta^{j}\right\| \leq C_{\star}\left(k^{p-1 / 2}+h^{r} k^{-1 / 2}\right) \leq 1 / 2,
$$

and for the last estimate to be satisfied we need to assume $k$ and $h^{2 r} k^{-1}$ to be sufficiently small; it is then easily seen that $U^{j} \in T_{u}, j=0, \ldots, n-1$. From (5.12), (5.14) and (5.17) the desired estimate (5.11) follows and the proof is complete.

For several examples of multistep schemes as well as for partial differential equations satisfying the conditions of this paper we refer the reader to [2] and [1.

Remark 5.1. If the estimate (1.3) holds in tubes around $u$ defined in terms of other norms, the mesh condition " $h^{2 r} k^{-1}$ small" of Theorem 5.1 has to be modified; see Remark 2.2 in [2].

\section{Computation of starting approximations}

In this section we present two schemes, one nonlinear and one linear, for the computation of starting approximations satisfying condition (5.10). We assume that $B(t, \cdot)$ can be modified to yield an operator $\mathcal{B}(t, \cdot): V \rightarrow V^{\prime}$ coinciding with $B(t, \cdot)$ in the tube $T_{u}, \mathcal{B}(t, \cdot)=B(t, \cdot)$ for all $v \in T_{u}$, and satisfying the global Lipschitz condition (cf. (1.3))

$$
\|\mathcal{B}(t, v)-\mathcal{B}(t, w)\|_{\star} \leq \lambda\|v-w\|+\mu|v-w| \quad \forall v, w \in V .
$$

Otherwise, for the nonlinear scheme the assumptions of Section 5 will be sufficient, while for the linear scheme some additional natural hypotheses will be needed. In particular, we assume that $k$ and $h^{2 r} k^{-1}$ are sufficiently small. 
A nonlinear scheme. Assume that the data of problem (1.1) are smooth enough such that one can determine the derivatives $u^{(j)}, j=0, \ldots, p-1$, of the solution at $t=0$. Let $\varphi^{m}$ be given by $\varphi^{m}:=u^{0}-\sum_{j=2}^{p-1} \frac{j-1}{j}(m k)^{j} u^{(j)}(0)$; these functions may be considered given since $u^{\prime}(0), \ldots, u^{(p-1)}(0)$ may be recursively determined from the data of (1.1).

Let $m \in\{1, \ldots, q-1\}$. It is easily seen that

$$
u\left(t^{m}\right)-m k u^{\prime}\left(t^{m}\right)=\varphi^{m}+\psi^{m},
$$

with $\psi^{m}$ such that

$$
\left|\psi^{m}\right| \leq C k^{p} .
$$

Using in (6.2) the differential equation of (1.1) and letting $u^{m}:=u\left(t^{m}\right)$, we obtain

$$
u^{m}+m k\left[A u^{m}-B\left(t^{m}, u^{m}\right)\right]=\varphi^{m}+\psi^{m}
$$

which motivates the definition of $U^{m} \in V_{h}$ by

$$
U^{m}+m k\left[A_{h} U^{m}-B_{h}\left(t^{m}, U^{m}\right)\right]=P_{o} \varphi^{m} .
$$

Besides this scheme, we also consider the modified scheme

$$
V^{m}+m k\left[A_{h} V^{m}-\mathcal{B}_{h}\left(t^{m}, V^{m}\right)\right]=P_{o} \varphi^{m}
$$

with $\mathcal{B}_{h}(t, \cdot): V \rightarrow V_{h}$ such that $\left(\mathcal{B}_{h}(t, \varphi), \chi\right)=(\mathcal{B}(t, \varphi), \chi)$ for all $\chi \in V_{h}$. We shall show that $V^{m}$ is well defined by $\left(6.5^{\prime}\right)$; then we will estimate $W^{m}-V^{m}$, and we will finally conclude that under our mesh condition $U^{m}$ is a locally unique solution of (6.5) satisfying (5.10).

Existence. To establish existence of $V^{m}$ we shall make use of the following version of the Brouwer fixed-point theorem.

Lemma 6.1. Let $(H,(\cdot, \cdot))$ be a finite dimensional inner product space and denote its norm by $|\cdot|$. Let $g: H \rightarrow H$ be continuous, and assume that, for some positive $\delta,(g(x), x)>0$ for all $x \in H$ such that $|x|=\delta$. Then, there exists an $x^{\star} \in H$ with $\left|x^{\star}\right|<\delta$ such that $g\left(x^{\star}\right)=0$.

To apply Lemma 6.1 to show the existence of a solution of $\left(6.5^{\prime}\right)$, let $g: V_{h} \rightarrow V_{h}$ be given by $g(v):=v+m k\left[A_{h} v-\mathcal{B}_{h}\left(t^{m}, v\right)\right]-P_{o} \varphi^{m}$. Obviously, $g$ is continuous. Furthermore,

$$
\begin{aligned}
(g(v), v) & =|v|^{2}+m k(A v, v)-m k\left(\mathcal{B}\left(t^{m}, v\right), v\right)-\left(\varphi^{m}, v\right) \\
& =|v|^{2}+m k\|v\|^{2}-m k\left(\mathcal{B}\left(t^{m}, v\right)-\mathcal{B}\left(t^{m}, 0\right), v\right)-\left(m k \mathcal{B}\left(t^{m}, 0\right)+\varphi^{m}, v\right) \\
& \geq|v|^{2}+m k\|v\|^{2}-m k\left\|\mathcal{B}\left(t^{m}, v\right)-\mathcal{B}\left(t^{m}, 0\right)\right\|\left\|_{\star}\right\| v \|-\left|m k \mathcal{B}\left(t^{m}, 0\right)+\varphi^{m}\right||v| .
\end{aligned}
$$

Now, using (6.1) and the algebraic-geometric inequality, we obtain

$$
(g(v), v) \geq \frac{1}{4}|v|^{2}+m k\left(1-\lambda-m k \mu^{2}\right)\|v\|^{2}-\frac{1}{2}\left|m k \mathcal{B}\left(t^{m}, 0\right)+\varphi^{m}\right|^{2},
$$

i.e., for $k$ sufficiently small such that $1-\lambda-m k \mu^{2} \geq 0$,

$$
(g(v), v) \geq \frac{1}{4}|v|^{2}-\frac{1}{2}\left|m k \mathcal{B}\left(t^{m}, 0\right)+\varphi^{m}\right|^{2} .
$$

Letting $v \in V_{h}$ in (6.6) be such that $|v|=\frac{1}{\sqrt{2}}\left|m k \mathcal{B}\left(t^{m}, 0\right)+\varphi^{m}\right|+1$, we have $(g(v), v)>0$, and we conclude, in view of Lemma 6.1, the existence of a solution $V^{m} \in V_{h}$ of $\left(6.5^{\prime}\right)$, provided $k$ is sufficiently small such that $1-\lambda-m k \mu^{2} \geq 0$. 
Uniqueness. Let $\tilde{V}^{m} \in V_{h}$ be such that

$$
\tilde{V}^{m}+m k\left[A_{h} \tilde{V}^{m}-\mathcal{B}_{h}\left(t^{m}, \tilde{V}^{m}\right)\right]=P_{o} \varphi^{m}
$$

Subtracting $\left(6.5^{\prime \prime}\right)$ from $\left(6.5^{\prime}\right)$ and letting $e^{m}:=V^{m}-\tilde{V}^{m}$, we obtain

$$
e^{m}+m k A_{h} e^{m}-m k\left[\mathcal{B}_{h}\left(t^{m}, V^{m}\right)-\mathcal{B}_{h}\left(t^{m}, \tilde{V}^{m}\right)\right]=0 .
$$

Taking here the inner product with $e^{m}$ and using (6.1), we have

$$
\left|e^{m}\right|^{2}+m k\left\|e^{m}\right\|^{2}-m k\left(\lambda\left\|e^{m}\right\|+\mu\left|e^{m}\right|\right)\left\|e^{m}\right\| \leq 0
$$

i.e.,

$$
\frac{1}{2}\left|e^{m}\right|^{2}+m k\left(1-\lambda-\frac{m k \mu^{2}}{2}\right)\left\|e^{m}\right\|^{2} \leq 0 .
$$

For $k$ sufficiently small such that $1-\lambda-\frac{m k \mu^{2}}{2} \geq 0$, we conclude from (6.7) that $e^{m}=0$, i.e., $\tilde{V}^{m}=V^{m}$.

Error estimates. To derive an estimate for $W^{m}-V^{m}$, we need an equation for $W^{m}$. For $\chi \in V_{h}$ we have

$$
\begin{aligned}
\left(W^{m}+\right. & \left.m k\left[A_{h} W^{m}-B_{h}\left(t^{m}, W^{m}\right)\right]-P_{o} \varphi^{m}, \chi\right) \\
= & \left(W^{m}, \chi\right)+m k\left(A W^{m}-B^{\prime}\left(t^{m}, u^{m}\right) W^{m}+\kappa W^{m}, \chi\right) \\
& -m k\left(B\left(t^{m}, W^{m}\right)-B^{\prime}\left(t^{m}, u^{m}\right) W^{m}+\kappa W^{m}-\varphi^{m}, \chi\right),
\end{aligned}
$$

i.e., in view of the definition of $R_{h}(t)$,

$$
\begin{aligned}
\left(W^{m}+\right. & \left.m k\left[A_{h} W^{m}-B_{h}\left(t^{m}, W^{m}\right)\right]-P_{o} \varphi^{m}, \chi\right) \\
= & \left(W^{m}, \chi\right)+m k\left(A u^{m}-B\left(t^{m}, u^{m}\right), \chi\right)+m k \kappa\left(u^{m}-W^{m}, \chi\right)-\left(\varphi^{m}, \chi\right) \\
& +m k\left(B\left(t^{m}, u^{m}\right)-B\left(t^{m}, W^{m}\right)-B^{\prime}\left(t^{m}, u^{m}\right)\left(u^{m}-W^{m}\right), \chi\right) .
\end{aligned}
$$

Using (6.4) here, we get

$$
\begin{aligned}
\left(W^{m}+\right. & \left.m k\left[A_{h} W^{m}-B_{h}\left(t^{m}, W^{m}\right)\right]-P_{o} \varphi^{m}, \chi\right) \\
= & \left(W^{m}-u^{m}, \chi\right)+\left(\psi^{m}, \chi\right)+m k \kappa\left(u^{m}-W^{m}, \chi\right) \\
& +m k\left(B\left(t^{m}, u^{m}\right)-B\left(t^{m}, W^{m}\right)-B^{\prime}\left(t^{m}, u^{m}\right)\left(u^{m}-W^{m}\right), \chi\right),
\end{aligned}
$$

i.e., in view of (5.3), (5.6) and (6.3),

$$
W^{m}+m k\left[A_{h} W^{m}-B_{h}\left(t^{m}, W^{m}\right)\right]=P_{o} \varphi^{m}+\zeta_{1}^{m}+k \zeta_{2}^{m}
$$

with

$$
\left|\zeta_{1}^{m}\right|+\left\|\zeta_{2}^{m}\right\|_{\star} \leq C\left(k^{p}+h^{r}\right) .
$$

Now let $\vartheta^{m}:=W^{m}-V^{m}$. Subtracting $\left(6.5^{\prime}\right)$ from (6.8), we obtain

$$
\vartheta^{m}+m k A_{h} \vartheta^{m}-m k\left[B_{h}\left(t^{m}, W^{m}\right)-\mathcal{B}_{h}\left(t^{m}, V^{m}\right)\right]=\zeta_{1}^{m}+k \zeta_{2}^{m} .
$$

Taking here the inner product with $\vartheta^{m}$ and using the fact that $W^{m} \in T_{u}$, we have

$$
\left|\vartheta^{m}\right|^{2}+m k\left\|\vartheta^{m}\right\|^{2}-m k\left(\mathcal{B}\left(t^{m}, W^{m}\right)-\mathcal{B}\left(t^{m}, U^{m}\right), \vartheta^{m}\right)=\left(\zeta_{1}^{m}, \vartheta^{m}\right)+k\left(\zeta_{2}^{m}, \vartheta^{m}\right),
$$

i.e., in view of (6.1),

$$
\begin{aligned}
\left|\vartheta^{m}\right|^{2} & +m k(1-\lambda)\left\|\vartheta^{m}\right\|^{2}-\mu m k\left|\vartheta^{m}\right|\left\|\vartheta^{m}\right\| \\
& \leq\left|\zeta_{1}^{m}\right|\left|\vartheta^{m}\right|+k\left\|\zeta_{2}^{m}\right\|_{\star}\left\|\vartheta^{m}\right\| .
\end{aligned}
$$

Now using the algebraic-geometric inequality, we obtain

$$
\frac{1}{4}\left|\vartheta^{m}\right|^{2}+k\left(m-\lambda m-\mu^{2} m^{2} k-\frac{1}{2} k\right)\left\|\vartheta^{m}\right\|^{2} \leq \frac{1}{2}\left|\zeta_{1}^{m}\right|^{2}+\frac{1}{2}\left\|\zeta_{2}^{m}\right\|_{\star}^{2} .
$$


Letting $k$ be sufficiently small here such that $m(1-\lambda)-\left(\mu^{2} m^{2}+\frac{1}{2}\right) k \geq c$ with a positive constant $c$, we easily obtain, in view of (6.9),

$$
\left|\vartheta^{m}\right|^{2}+k\left\|\vartheta^{m}\right\|^{2} \leq C\left(k^{p}+h^{r}\right)^{2} .
$$

In view of our mesh condition, it easily follows from (6.10) and (5.3) that $V^{m} \in T_{u}$; thus $V^{m}$ is also a locally unique solution of (6.5). Furthermore, obviously, if (6.10) is satisfied for $m=1, \ldots, q-1$ (and $U^{0}=W^{0}$ ), then (5.10) is also satisfied.

A linear scheme. In the analysis of the linear scheme we will present in the sequel, besides (6.1) and the hypotheses of Section 5, we shall assume three additional natural conditions, two for the nonlinearity $B$, namely that

$$
\left\|B(t, v)-B(t, w)-B^{\prime}(t, w)(v-w)\right\|_{\star} \leq C\|v-w\|^{2} \quad \forall v, w \in T_{u}
$$

and that the operator $A-B^{\prime}(t, v)+\kappa I$ is, for an appropriate constant $\kappa$ and $v \in T_{u}$, positive definite, uniformly in $t$ and $v$ (cf. the hypothesis on $M(t)$ in Section 5) and one analog to (5.3). To formulate the third condition, we let $R_{h}: V \rightarrow V_{h}$ denote an "elliptic" projection defined, in terms of the linear operator $A$ only, by

$$
P_{o} A R_{h} v=P_{o} A v ;
$$

cf. the definition of $R_{h}(t)$ in Section 5. The assumption then is that $R_{h}$ satisfies the estimate

$$
\left\|u(t)-R_{h} u(t)\right\| \leq C h^{r-\frac{d}{2}}
$$

cf. (5.3).

We shall linearize the scheme (6.5) by Newton's method and we will show that, for appropriate starting approximations $U_{0}^{m}$, one Newton iteration suffices to obtain initial approximations $U_{1}^{m}$ satisfying (5.10).

We begin with the definition of an appropriate starting approximation $U_{0}^{m}$. Let $T_{m}^{p} u(0)$ be given by

$$
T_{m}^{p} u(0):=u^{0}+m k u^{\prime}(0)+\cdots+\frac{(m k)^{p-1}}{(p-1) !} u^{(p-1)}(0), \quad m=1, \ldots, q-1 .
$$

We then let $U_{0}^{m}:=R_{h} T_{m}^{p} u(0) \in V_{h}$-notice that $R_{h}\left(t^{m}\right) T_{m}^{p} u(0)$ cannot be computed since the solution at $t^{m}$, and consequently $M\left(t^{m}\right)$, is not known-i.e., $U_{0}^{m}$ is given by

$$
\left(A_{h} U_{0}^{m}, \chi\right)=\left(A T_{m}^{p} u(0), \chi\right) \quad \forall \chi \in V_{h} .
$$

To estimate $U^{m}-U_{0}^{m}$, we let $\tilde{W}^{m}:=R_{h} u^{m}$, i.e., $\tilde{W}^{m}$ is given by

$$
\left(A_{h} \tilde{W}^{m}, \chi\right)=\left(A u^{m}, \chi\right) \quad \forall \chi \in V_{h} .
$$

Subtracting (6.14) from (6.15), we obtain

$$
\left(A_{h}\left(\tilde{W}^{m}-U_{0}^{m}\right), \chi\right)=\left(A\left(u^{m}-T_{m}^{p} u(0)\right), \chi\right) \quad \forall \chi \in V_{h} .
$$

Letting $\chi:=\tilde{W}^{m}-U_{0}^{m}$ here, we get

$$
\left\|\tilde{W}^{m}-U_{0}^{m}\right\| \leq\left\|u^{m}-T_{m}^{p} u(0)\right\|,
$$

and we conclude that

$$
\left\|\tilde{W}^{m}-U_{0}^{m}\right\| \leq C k^{p} .
$$

Let us notice here for later use that, in view of (6.13) and (6.16), $U_{0}^{m} \in T_{u}$. 
Furthermore,

$$
U^{m}-U_{0}^{m}=\left(U^{m}-W^{m}\right)+\left(W^{m}-u^{m}\right)+\left(u^{m}-\tilde{W}^{m}\right)+\left(\tilde{W}^{m}-U_{0}^{m}\right) ;
$$

since, according to (5.3) and (6.13),

$$
\left\|W^{m}-u^{m}\right\|+\left\|u^{m}-\tilde{W}^{m}\right\| \leq C h^{r-\frac{d}{2}},
$$

in view of (6.10) and (6.16) we get

$$
\left\|U^{m}-U_{0}^{m}\right\| \leq C k^{-\frac{1}{2}}\left(k^{p}+h^{r}\right)+C\left(k^{p}+h^{r-\frac{d}{2}}\right),
$$

i.e.,

$$
\left\|U^{m}-U_{0}^{m}\right\| \leq C\left(k^{p-\frac{1}{2}}+h^{r} k^{-\frac{1}{2}}+h^{r-\frac{d}{2}}\right) .
$$

Starting with $U_{0}^{m}$, we let $U_{1}^{m} \in V_{h}$ be an approximation to $U^{m}$ given by performing one iteration with Newton's method applied to (6.5), i.e., $U_{1}^{m}$ is given by

$$
\begin{aligned}
{\left[I+m k A_{h}\right.} & \left.-m k B_{h}^{\prime}\left(t^{m}, U_{0}^{m}\right)\right]\left(U_{1}^{m}-U_{0}^{m}\right) \\
& +U_{0}^{m}+m k A_{h} U_{0}^{m}-m k B_{h}\left(t^{m}, U_{0}^{m}\right)=P_{o} \varphi^{m}
\end{aligned}
$$

which can be written in the form

$$
\begin{aligned}
{\left[I+m k A_{h}\right.} & \left.-m k B_{h}^{\prime}\left(t^{m}, U_{0}^{m}\right)\right] U_{1}^{m} \\
& =m k B_{h}\left(t^{m}, U_{0}^{m}\right)+m k B_{h}^{\prime}\left(t^{m}, U_{0}^{m}\right) U_{0}^{m}+P_{o} \varphi^{m} .
\end{aligned}
$$

Using the fact that $U_{0}^{m} \in T_{u}$ and the assumption that $A-B^{\prime}\left(t, U_{0}^{m}\right)+\kappa I$ is positive definite, we easily see that $U_{1}^{m}$ is well defined for $k<\frac{1}{\kappa m}$.

Now let $e_{i}^{m}:=U^{m}-U_{i}^{m}$. Subtracting (6.18) from (6.5), we obtain

$$
\begin{aligned}
e_{1}^{m}+m k A_{h} e_{1}^{m} & -m k B_{h}^{\prime}\left(t^{m}, U_{0}^{m}\right) e_{1}^{m} \\
& =m k\left[B_{h}\left(t^{m}, U^{m}\right)-B_{h}\left(t^{m}, U_{0}^{m}\right)-B_{h}^{\prime}\left(t^{m}, U_{0}^{m}\right)\left(U^{m}-U_{0}^{m}\right)\right],
\end{aligned}
$$

i.e.,

$$
\begin{aligned}
& (1-\kappa m k) e_{1}^{m}+m k\left[A_{h}-B_{h}^{\prime}\left(t^{m}, U_{0}^{m}\right)+\kappa I\right] e_{1}^{m} \\
& \quad=m k\left[B_{h}\left(t^{m}, U^{m}\right)-B_{h}\left(t^{m}, U_{0}^{m}\right)-B_{h}^{\prime}\left(t^{m}, U_{0}^{m}\right)\left(U^{m}-U_{0}^{m}\right)\right] .
\end{aligned}
$$

Taking here the inner product with $e_{1}^{m}$ and using the fact that $A-B^{\prime}(t, v)+\kappa I$ is positive definite, uniformly in $t$ and $v, v \in T_{u}$, and $U^{m}, U_{0}^{m} \in T_{u}$, and (6.11), we get

$$
(1-\kappa m k)\left|e_{1}^{m}\right|^{2}+c m k\left\|e_{1}^{m}\right\|^{2} \leq C k\left\|e_{0}^{m}\right\|^{2}\left\|e_{1}^{m}\right\|,
$$

with a positive constant $c$, i.e., for $k<\frac{1}{2 \kappa m}$,

$$
\left|e_{1}^{m}\right|^{2}+k\left\|e_{1}^{m}\right\|^{2} \leq C k\left\|e_{0}^{m}\right\|^{4} .
$$

From (6.20) and (6.17) we obtain

$$
\left|e_{1}^{m}\right|^{2}+k\left\|e_{1}^{m}\right\|^{2} \leq C k\left(k^{4 p-2}+h^{4 r} k^{-2}+h^{4 r-2 d}\right),
$$

i.e.,

and thus

$$
\left|e_{1}^{m}\right|^{2}+k\left\|e_{1}^{m}\right\|^{2} \leq C\left(k^{2 p}+h^{4 r} k^{-1}+h^{2 r}\right)
$$

$$
\left|e_{1}^{m}\right|^{2}+k\left\|e_{1}^{m}\right\|^{2} \leq C\left(k^{2 p}+h^{2 r}\right),
$$

i.e.,

$$
\left|e_{1}^{m}\right|^{2}+k\left\|e_{1}^{m}\right\|^{2} \leq C\left(k^{p}+h^{r}\right)^{2} .
$$


From (6.10) and (6.21) we finally conclude

$$
\left|U_{1}^{m}-W^{m}\right|+\sqrt{k}\left\|U_{1}^{m}-W^{m}\right\| \leq C\left(k^{p}+h^{r}\right) .
$$

Remark 6.1. Condition (6.11) can easily be relaxed or modified if one is willing to perform more iterations with Newton's method in order to obtain starting approximations satisfying the analog of (6.22).

\section{Combination of implicit Runge-Kutta schemes And EXtrapolation}

Keeling [5] constructs and analyzes implicit-explicit schemes for semilinear parabolic equations. The linear part of the equation is discretized by implicit RungeKutta methods (IRKM) and the nonlinear part by extrapolation from previous time levels. One advantage of the resulting implicit-explicit schemes is that their polynomial order coincides with their order and hence, in contrast to IRKM, they do not suffer from order reduction; a computational advantage of these schemes is that to advance in time linear problems with the same operator for all time levels have to be solved. We will see in the sequel that Keeling's schemes applied to (1.1) are particular cases of the schemes (1.2). Let us note that in this particular case the results of this paper improve those of [5] in various aspects: we allow stronger nonlinearities, and in particular we have an optimal bound for the stability constant $\lambda$, and we also get by with milder mesh conditions; cf. also [2] and [1] for concrete parabolic equations.

In this section we will focus on single step schemes for the discretization of the linear part of equation (1.1). Thus we let $\rho_{q}=1, \rho_{q-1}=-\rho$, and $\rho_{i}=0, i=$ $0, \ldots, q-2$. It is then easily seen that the strong $A(0)$-stability condition of a consistent scheme described by $\left(\rho_{0}, \ldots, \rho_{q}\right)$ reads in this case

$$
\rho(0)=1, \quad \rho^{\prime}(0)<0, \quad|\rho(x)|<1 \text { for } x \in(0, \infty] .
$$

Given an integer $r \geq 1$, an $r$-stage IRKM is characterized by a set of constants arranged in tableau form

\begin{tabular}{l|l}
$\mathcal{Q}$ & $\tau$ \\
\hline$b^{T}$ &
\end{tabular}

with $\mathcal{Q}=\left(a_{i j}\right) \in \mathbb{R}^{r, r}, b=\left(b_{1}, \ldots, b_{r}\right)^{T}$ and $\tau=\left(\tau_{1}, \ldots, \tau_{r}\right)^{T}$. Let $p \geq 1$ be the order of the IRKM; actually we will only need the relations

$$
b^{T} \mathcal{O}^{\ell-1} e=\frac{1}{\ell !}, \quad \ell=1, \ldots, p,
$$

with $e:=(1, \ldots, 1)^{T}$. For stability purposes, we assume that the IRKM is strongly $A_{0}$-stable, i.e., for the corresponding rational approximation $\rho, \rho(z):=1-$ $z b^{T}(I+z \mathcal{Q})^{-1} e$, to the exponential $\mathrm{e}^{-z}$ it holds that

$$
\sup _{x \geq x_{0}}|\rho(x)|<1 \quad \forall x_{0}>0 .
$$

Let us note that, for $p \geq 1,(7.3)$ is equivalent to (7.1). Furthermore, we assume that the eigenvalues of $\mathcal{O}$ have nonnegative real parts, and $\mathcal{O}$ is invertible, i.e., $\sigma(\mathcal{O}) \subset\{z \in \mathbb{C}: \operatorname{Re} z \geq 0, z \neq 0\}$, with $\sigma(\mathcal{O})$ the spectrum of $\mathcal{O}$. 
Following Keeling, we define the extrapolation associated with an $r$-stage IRKM of order $p$ in terms of the constants $\alpha_{i j}, i=1, \ldots, r, j=0, \ldots, p-1$, given by

$$
\sum_{j=0}^{p-1} j^{\ell} \alpha_{i j}=(-1)^{\ell} \ell ! \hat{e}_{i}^{T} \mathcal{Q}^{\ell} e, \quad \ell=0, \ldots, p-1, i=1, \ldots, r,
$$

with $\hat{e}_{i} \in \mathbb{R}^{r},\left(\hat{e}_{i}\right)_{j}=\delta_{i j}$.

Suppose that approximations $U^{m}$ to $u^{m}, m=0, \ldots, n$, have been computed. We introduce the extrapolation operator $\mathcal{E}^{n}: V^{p} \rightarrow\left(V^{\prime}\right)^{r}, n \geq p-1$, by

$$
\left(\mathcal{E}^{n} \tilde{\mathbf{v}}^{n}\right)_{i}:=\sum_{j=0}^{p-1} \alpha_{i j} B\left(t^{n-j}, v^{n-j}\right), \quad i=1, \ldots, r,
$$

with $\tilde{\mathbf{v}}^{n}:=\left(v^{n}, \ldots, v^{n-p+1}\right)^{T}$. Also let $\mathcal{A}:(D(A))^{r} \rightarrow H^{r}, \mathcal{A}:=\operatorname{diag}(A, \ldots, A)$.

Now, Keeling's scheme applied to (1.1) yields $U^{n+1} \approx u^{n+1}, n \geq p-1$, defined by

$$
\begin{aligned}
\mathbf{U}^{n} & =U^{n} e-k \mathcal{O A} \mathcal{A} \mathbf{U}^{n}+k \mathcal{O} \mathcal{E}^{n} \tilde{\mathbf{U}}^{n}, \\
U^{n+1} & =\left(I-b^{T} \mathcal{Q}^{-1} e\right) U^{n}+b^{T} \mathcal{O}^{-1} \mathbf{U}^{n},
\end{aligned}
$$

with $\mathbf{U}^{n}=\left(U^{n, 1}, \ldots, U^{n, r}\right)^{T}$. We will write this scheme in the form of $(1.2)$ and will show that its order and polynomial order are $p$. Then, the results of the previous sections apply. First, (7.6) can be written in the form

$$
\begin{aligned}
U^{n+1} & =\left(I-k \mathcal{A} b^{T}(I+k \mathcal{O} \mathcal{A})^{-1} e\right) U^{n}+k b^{T}(I+k \mathcal{O A})^{-1} \mathcal{E}^{n} \tilde{\mathbf{U}}^{n} \\
& =\rho(k A) U^{n}+k b^{T}(I+k \mathcal{O} \mathcal{A})^{-1} \mathcal{E}^{n} \tilde{\mathbf{U}}^{n} \\
& =\rho(k A) U^{n}+k b^{T}(I+k \mathcal{O} \mathcal{A})^{-1} \sum_{i=0}^{p-1} \alpha^{i} B\left(t^{n-i}, U^{n-i}\right)
\end{aligned}
$$

with $\alpha^{i}:=\left(\alpha_{1 i}, \ldots, \alpha_{r i}\right)^{T}$. Therefore,

$$
U^{n+p}=\rho(k A) U^{n+p-1}+k b^{T}(I+k \mathcal{O A})^{-1} \sum_{i=0}^{p-1} \alpha^{i} B\left(t^{n+p-1-i}, U^{n+p-1-i}\right),
$$

i.e.,

$$
U^{n+p}=\rho(k A) U^{n+p-1}+k b^{T}(I+k \mathcal{O A})^{-1} \sum_{i=0}^{p-1} \alpha^{p-1-i} B\left(t^{n+i}, U^{n+i}\right) .
$$

With $\sigma_{i}(x):=b^{T}(I+x \mathcal{O})^{-1} \alpha^{p-1-i}, i=0, \ldots, p-1,(7.7)$ can be written as

$$
U^{n+p}-\rho(k A) U^{n+p-1}=k \sum_{i=0}^{p-1} \sigma_{i}(k A) B\left(t^{n+i}, U^{n+i}\right),
$$

which is of the form (1.2) with $q=p$.

Next, let us show that the polynomial order of the scheme (7.8) is $p$. First, let us rewrite (7.4): Let $\mathfrak{A}$ denote the $p \times r$ matrix with entries $\alpha_{i j}, f_{\ell}:=$ $\left(0,1^{\ell}, \ldots,(p-1)^{\ell}\right)^{T}$, and $f_{0}:=(1, \ldots, 1)^{T}$. Then, (7.4) can be written in the form

$$
\mathfrak{A} f_{\ell}=(-1)^{\ell} \ell ! \mathcal{Q}^{\ell} e, \quad \ell=0,1, \ldots, p-1 .
$$

Therefore,

$$
-\ell \mathfrak{A} f_{\ell-1}+x \mathfrak{A} f_{\ell}=(-1)^{\ell} \ell !(I+x \mathcal{O}) \mathcal{O}^{\ell-1} e,
$$


and, using the notation of Section 1,

$$
\begin{aligned}
\varphi_{\ell, p}(x) & =E_{0}\left(1, x, v_{\ell, p}\right) \\
& =1-(-1)^{\ell} b^{T}(I+x \mathcal{O})^{-1}\left(-\ell \mathfrak{A} f_{\ell-1}+x \mathfrak{A} f_{\ell}\right) \\
& =1-\ell ! b^{T} \mathcal{Q}^{\ell-1} e .
\end{aligned}
$$

Using (7.2) here, we see that $\varphi_{\ell, p}=0$ for $1 \leq \ell \leq p-1$. For $\ell=0$, since $v_{0, p}=1$ and $\mathfrak{A} f_{0}=e$, we have

$$
\varphi_{0, p}(x)=E_{0}\left(1, x, v_{0, p}\right)=1-\rho(x)-x b^{T}(I+x \mathcal{O})^{-1} e=0 .
$$

Hence, in view of $\left(\tilde{C}_{\tilde{p}}^{\prime}\right)$, the polynomial order of the method is $p$.

Similarly, we have $\varphi_{p, p}(0)=0$, and thus $\varphi_{p, p}(x)=O(x)$; consequently $\varphi_{p}(x)=$ $O(x)$, and we conclude that the order of the scheme is $p$.

Let us also note that letting $q=p$, letting the $\rho_{i}$ 's be as in the beginning of this section and determining the $\sigma_{i}$ 's from $(1.12)$ is an alternative way leading to Keeling's schemes.

Remark 7.1. The strong $A(0)$-stability condition cannot be relaxed to $A(0)$-stability, because then $K_{(\rho, \sigma)}$ may be infinite and, consequently, our stability condition (1.5) may deteriorate. We demonstrate this with an example. Let us consider the one-stage Gauss-Legendre Runge-Kutta method, i.e., the midpoint scheme; the scheme is described by the tableau

$$
\begin{array}{l|l}
\frac{1}{2} & \frac{1}{2} \\
\hline 1 &
\end{array}
$$

and the corresponding rational approximation $\rho$ to the exponential is given by $\rho(x)=\left(1-\frac{x}{2}\right) /\left(1+\frac{x}{2}\right)$. The order of the method is two, and according to (7.4) we have $\alpha_{10}=3 / 2, \alpha_{11}=-1 / 2$. Therefore

$$
\sigma_{0}(x)=-\frac{1}{2} \frac{1}{1+\frac{x}{2}}, \quad \sigma_{1}(x)=\frac{3}{2} \frac{1}{1+\frac{x}{2}} .
$$

Thus, we choose $q=2$, and the implicit scheme is described be the rational functions $\left(\rho_{0}, \rho_{1}, \rho_{2}\right)$ with $\rho_{0}=0, \rho_{1}=-\rho, \rho_{2}=1$. In this case we have

$$
\rho(x, \zeta)=\frac{\zeta}{1+\frac{x}{2}}\left(-\left(1-\frac{x}{2}\right)+\left(1+\frac{x}{2}\right) \zeta\right), \quad \sigma(x, \zeta)=\frac{1}{2\left(1+\frac{x}{2}\right)}(-1+3 \zeta) .
$$

Hence,

and thus $K_{(\rho, \sigma)}=\infty$.

$$
\max _{\zeta \in S_{1}}\left|\frac{x \sigma(x, \zeta)}{\rho(x, \zeta)}\right| \geq\left|\frac{x \sigma(x,-1)}{\rho(x,-1)}\right|=x
$$

Remark 7.2. Assume that $\rho_{0}, \ldots, \rho_{q-1}$ vanish at infinity; this means in the case of implicit multistep schemes that $\beta_{0}=\cdots=\beta_{q-1}=0$, which holds for the BDF schemes of order up to six, and for IRKM that the corresponding rational approximation $\rho$ vanishes at infinity. Then, the argument showing $K_{2}<\infty$ also shows that $\tilde{N}_{1}, \tilde{N}_{1}=\max _{0 \leq j \leq q-1} \sup _{x>0} \int_{0}^{1} x\left|\delta_{j}\left(\mathrm{e}^{-2 i \pi t}, x\right)\right|^{2} d t$, is finite. As a consequence, $(2.7 \mathrm{i})$ can be replaced by

$$
k \sum_{\ell=q}^{n}\left\|\vartheta_{2}^{\ell}\right\|^{2} \leq q \tilde{N}_{1} \sum_{j=0}^{q-1}\left|\vartheta_{2}^{j}\right|^{2} .
$$


Assume now that (1.3) is satisfied with $\lambda=0$. Then, in view of $\left(2.7^{\prime} \mathrm{i}\right)$, our analysis yields

$$
\left|\vartheta^{n}\right|^{2}+k \sum_{\ell=q}^{n}\left\|\vartheta^{\ell}\right\|^{2} \leq C \mathrm{e}^{c \mu^{2} t^{n}}\left\{\sum_{j=0}^{q-1}\left|\vartheta^{j}\right|^{2}+k \sum_{\ell=0}^{n-q}\left\|E_{h}\left(t^{\ell}\right)\right\|_{\star}^{2}\right\}
$$

instead of (5.16); this leads to the error estimate (5.11) under the following relaxed hypothesis on the starting approximations

$$
\max _{0 \leq j \leq q-1}\left|W^{j}-U^{j}\right| \leq C\left(k^{p}+h^{r}\right) \quad \text { and } \quad U^{0}, \ldots, U^{q-1} \in T_{u} .
$$

Let us also note that conversely $\tilde{N}_{1}<\infty$ implies that $\rho_{0}, \ldots, \rho_{q-1}$ vanish at infinity. Consequently, an inequality of the form $\left(2.7^{\prime} \mathrm{i}\right)$ with a finite constant is only valid for a subclass of the schemes considered in this paper.

\section{REFERENCES}

1. G. Akrivis, M. Crouzeix and Ch. Makridakis, Implicit-explicit multistep finite element methods for nonlinear parabolic problems, Math. Comp. 67 (1998) 457-477. MR 98q:65088

2. G. Akrivis, M. Crouzeix and Ch. Makridakis, Implicit-explicit multistep methods for quasilinear parabolic equations, Numer. Math. 82 (1999) 521-541. MR 2000e:65075

3. Ph. Brenner, M. Crouzeix and V. Thomée, Single step methods for inhomogeneous linear differential equations in Banach space, RAIRO Anal. Numér. 16 (1982) 5-26. MR 83d:65268

4. M. Crouzeix, Sur l'approximation des équations différentielles opérationnelles linéaires par des méthodes de Runge-Kutta. Thèse, Université de Paris VI, 1975.

5. S. L. Keeling, Galerkin/Runge-Kutta discretizations for semilinear parabolic equations, SIAM J. Numer. Anal. 27 (1990) 394-418. MR 91d:65140

6. J. Lang, Adaptive Multilevel Solution of Nonlinear Parabolic PDE Systems. Theory, Algorithm, and Applications. Lecture Notes in Computational Science and Engineering, v. 16, Springer-Verlag, Berlin, 2001. MR 2001i:65106

7. C. Lubich, On the convergence of multistep methods for nonlinear stiff differential equations, Numer. Math. 58 (1991) 839-853. MR 92d:65127

8. C. Lubich and A. Ostermann, Linearly implicit time discretization of non-linear parabolic equations, IMA J. Numer. Anal. 15 (1995) 555-583. MR 96q:65085

9. G. Savaré, $A(\theta)$-stable approximations of abstract Cauchy problems, Numer. Math. 65 (1993) 319-335. MR 94h:65062

10. T. Steihaug and A. Wolbrandt, An attempt to avoid exact Jacobian and nonlinear equations in the numerical solution of stiff differential equations, Math. Comp. 33 (1979) 521-534. MR 80q:65087

11. V. Thomée, Galerkin Finite Element Methods for Parabolic Problems. Springer-Verlag, Berlin, 1997. MR 86k:65006

Computer Science Department, University of Ioannina, 45110 Ioannina, Greece

E-mail address: akrivis@cs.uoi.gr

IRMaR, Université de Rennes I, Campus de Beaulieu, F-35042 Rennes, France

E-mail address: michel.crouzeix@univ-rennes1.fr 